\title{
Microbubble Enhanced Ultrasound-Assisted Catheter-Directed Thrombolysis for VTE- A Sound Idea?
}

\author{
Andrew S. P. Sharp ${ }^{1}$ \\ ${ }^{1}$ Royal Devon and Exeter Hospital and University of Exeter, Exeter, \\ Devon, United Kingdom \\ Thromb Haemost 2019;119:1036.
}

Venous thromboembolism (VTE) is the third most common cause of cardiovascular death in Europe and a major cause of morbidity. ${ }^{1}$

The vast majority of patients presenting with proximal deep vein thrombosis or pulmonary embolism $(\mathrm{PE})$ are treated with anticoagulation, a low-risk, but sometimes ineffective option, with up to 1 in 5 of patients with intermediate high-risk PE experiencing cardiovascular collapse or death in the Pulmonary Embolism Thrombolysis trial, when tachycardia, hypoxia, and/or relative hypotension were present. ${ }^{2}$

Catheter-directed thrombolysis (CDT) for VTE is an old concept, although the lack of consistency of approach and hard outcome data are sometimes criticized. First, however, a robust protocol for $\mathrm{CDT}$ is required.

In $\mathrm{PE}$, a simple pigtail catheter could clearly deliver thrombolytic to the pulmonary artery in sufficient quantities that clot dissolution could occur, but shunting into unobstructed pulmonary arteries might mean limited benefit of this approach over and above that of systemic thrombolysis.

Ultrasound-assisted CDT is designed to enhance CDT by augmenting local delivery through acoustic streaming of the drug and the thinning of fibrin strands. ${ }^{3}$

The article by Engelberger et al suggests that ultrasound does indeed augment thrombolysis in a human clot in vitro model. ${ }^{4}$ Well-conducted, investigator-led data supporting a potential benefit from ultrasound augmentation are welcome.

The authors go further and use Sonovue bubble contrast media, widely available in echocardiography laboratories around the world, to produce microbubble cavitationassisted drug delivery and enhance clot dissolution properties when used in conjunction with ultrasound.

Showing an increase in d-dimer levels (suggesting greater breakdown of fibrin) and incrementally increasing clot
Address for correspondence Andrew S. P. Sharp, MBChB, MD, FRCP, Royal Devon and Exeter Hospital and University of Exeter, Barrack Road Exeter Devon EX2 5DW, United Kingdom (e-mail: drandrewsharp@gmail.com).

weight reduction with the addition of ultrasound and then ultrasound with microbubbles, the two CDT treatment adaptations appear to be synergistic for clot dissolution.

Whether this bench model will translate into clinical benefit when tested within the more mature clot sometimes found in clinical VTE, and whether the more complex flow dynamics of a human pulmonary arterial system will prove a more complex challenge for microbubble augmentation than a bench test, remains to be seen, but the next step would seem to be an in vivo study and then clinical outcome data.

In an area in need of more hard outcome data, consensus on an optimal CDT protocol and then a well-designed multicenter clinical outcomes trial would be welcome in this area of significant unmet clinical need.

Conflict of Interest

A.S.P.S is a consultant for BTG and reports research funding from BTG.

\section{References}

1 Cohen AT, Agnelli G, Anderson FA, et al; VTE Impact Assessment Group in Europe (VITAE). Venous thromboembolism (VTE) in Europe. The number of VTE events and associated morbidity and mortality. Thromb Haemost 2007;98(04):756-764

2 Barco S, Vicaut E, Klok FA, Lankeit M, Meyer G, Konstantinides SV; PEITHO Investigators. Improved identification of thrombolysis candidates amongst intermediate-risk pulmonary embolism patients: implications for future trials. Eur Respir J 2018;51(01):1701775

3 Francis CW, Blinc A, Lee S, Cox C. Ultrasound accelerates transport of recombinant tissue plasminogen activator into clots. Ultrasound Med Biol 1995;21(03):419-424

4 Engelberger RP, Schroeder V, Nagler M, et al. Enhanced thrombolysis by ultrasound-assisted catheter-directed thrombolysis and microbubbles in an in vitro model of iliofemoral deep vein thrombosis. Thromb Haemost 2019;119(07):1094-1101 received

May 28, 2019

accepted after revision

May 28, 2019 (c) 2019 Georg Thieme Verlag KG Stuttgart · New York
DOI https://doi.org/

10.1055/s-0039-1692644. ISSN 0340-6245. 